\title{
Application Research of Block-Chain Technology in Intelligent Logistics Tracking System
}

\author{
Liucheng Zhang \\ School of Accounting, Harbin University of Commerce, Harbin, 150028, China \\ zhagnliucheng@126.com
}

Keywords: Blockchain, Logistics, Logistics tracking

\begin{abstract}
With the development of blockchain, blockchain technology has become increasingly mature in recent years, and its digitalization features such as decentralization, trust, openness, transparency, and non-tampering make blockchain technology in various fields. It has received extensive attention, and logistics and supply chain are considered to be one of the industries that are most helpful for improvement. This paper discusses the principle and characteristics of the blockchain. By analyzing the development status of the blockchain technology and the problems in the development of China's logistics express industry, exploring the application of blockchain technology in the field of logistics. Finally, through summarization and analysis, explore the application of blockchain technology in the creation of intelligent logistics tracking system, so as to provide guarantee for the development of logistics, so that it can better solve the problems of logistics transportation and distribution, parcel settlement, etc., and make the package information more Clearly improve the security of information.
\end{abstract}

\section{Introduction}

After the concept of "Bitcoin" was proposed by Nakamoto in 2008, the digital cryptocurrency system represented by Bitcoin began to enter the financial and other fields, and the blockchain came into being ${ }^{[1]}$. Under the rapid development of "Internet + " and blockchain technology, many people regard blockchain technology as an important engine of the fourth industrial revolution after the steam age, electrical age and information age. In the logistics express industry, "blockchain+logistics" has also become an important exploration for the future development of the logistics field.

\section{The concept and characteristics of blockchain technology}

\subsection{Blockchain Definition.}

Blockchain technology is a new technology that has been proposed in recent years. It is still in the stage of exploration and development, so it has not yet formed a unified concept. In the book "Blockchain: The New Economic Blueprint and Guide" American scholar Melanie Sw an gives the following definition of blockchain: blockchain technology is an open and transparent, decentralized database. In addition, in the book "China Blockchain Technology and Industrial Development White Paper" written by China Blockchain Technology and Industry Development Forum, the blockchain is defined as: distributed data storage, point-to-point transmission, consensus mechanism, A new application model for computer technologies such as encryption algorithms.

\subsection{Characteristics of blockchain technology.}

Blockchain technology, as a kind of distributed transaction book, mainly adopts blockchain data structure, distributed node consensus algorithm and cryptography principle, which makes it decentralized, trusted, open and transparent, and cannot be falsified. And traceability and other features. Blockchains are generally divided into three chain structures: public chain, alliance chain and private chain, depending on their application scenarios and design systems. 
A public blockchain means that any organization or individual in the world can apply for joining a blockchain and conduct transactions, and the transaction can obtain valid confirmation of the blockchain, and anyone can participate in the consensus process. The earliest public blockchains appear in these three major chains are also the most widely used blockchains.

The alliance blockchain refers to a blockchain composed of multiple organizations for a common goal. Some related organizations can join the alliance chain after seeking the consent of the alliance. This form of blockchain will become the mainstream of future blockchains.

The private blockchain refers to the accounting function only through the general ledger technology of the blockchain. It is a blockchain that specializes in serving an organization or a simple business. Therefore, it can be a company or an individual unit. Write permission for the blockchain. Therefore, the private chain has a large degree of closure and exclusivity, usually implemented in a small scope, and because its target is single, the construction is relatively simple.

\section{The development status of blockchain}

\subsection{Development of the world's blockchain.}

Since 2012, the development trend of global blockchain has been very rapid, which has attracted worldwide attention. At present, the most rapid development of blockchain is still in the United States and the United Kingdom. On January 19, 2016, the British government issued an important report "distributed ledger technology: beyond blockchain", aiming to explore the ledger technology similar to blockchain, which is used to record the ownership of goods and intellectual property, and protect government privacy, etc. [5]. The development of blockchain has received more and more attention.

\subsection{Development of China's blockchain.}

At present, blockchain has also received high attention in Chinese industry. Jingdong, alibaba and other enterprises have been the first to conduct experimental research on blockchain technology. The number of new enterprises in China's blockchain has increased significantly since 2014, mainly in first-tier cities such as Beijing, Shanghai and Guangzhou. According to the current development trend of blockchain, "blockchain + logistics" will be a new breakthrough in the development of China's logistics industry.

\section{Exploration of application of blockchain technology in the field of logistics}

\subsection{Application of blockchain technology in logistics transportation.}

In logistics transportation, it is possible to create a blockchain system exclusive to logistics transportation based on the block chain technology to realize intelligent transportation. We can use the block chain technology, according to the movement of goods region, a first for automatic classification category, then intelligent arrange cargo transportation route and arrange shipping schedule, with the aid of block chain technology for transport of goods before, after transportation, transportation in the whole process chain block, record the goods from issued to the user to sign for all process node. All participants in the blockchain system can record and store changes in the data information of the goods in case of changes in the delivery status during the transportation. The entire transport process of goods is open and transparent to all participants in the system, ensuring that all people can timely track the logistics information of goods. To make the logistics information more clear to prevent the occurrence of the false claim of the parcel and the occurrence of the problem of missing parts and explosive storehouse. In this way, not only the work efficiency of the whole department is improved, but also every transportation link can be monitored and inquired, so that users and enterprises can trace the logistics information and capital of goods.

\subsection{Application of block chain technology in logistics supply chain industry.}

In the supply chain industry, logistics, capital flow, information flow and many other entities are 
often involved. There are a lot of complex cooperation and communication between these entities. Between different entities in traditional mode, because it is kept by their respective supply chain information, information data is the lack of certain safety and transparency, and the corresponding caused high time cost and the cost of capital, then once a link problems such as wrong by mistake maxillary and lost, will be difficult to trace and processing. If the blockchain technology is used, all parties can conduct transactions on the same safe and reliable information platform and ensure that all participants can check the status of goods at any time, which not only reduces the logistics cost, but also conveniently tracks the entire production and delivery process of goods. In the event of a dispute, forensics and tracing would be easy. On the other hand, new supply chain solutions based on blockchain technology can be created to synchronize the flow of goods and capital and alleviate the problem of fake goods.

\subsection{Application of blockchain technology in logistics trading platform.}

Along with the rise of e-commerce and online shopping, the most of the information interaction is with the aid of trading platform, logistics, express industry is also on trading platform and running, it is to meet the demand in transport of the goods, to ensure that the goods can not restricted by time and region under the condition of distribution, and in the standard time arrived. And block the application of chain technology is to need to rely on the corresponding database implementation, block chain are applied to the logistics information platform, by means of block chain technology to the center, to trust, and the characteristics of the whole people to participate in an account, you can solve the commodity trading link in the process of trading faces information opaque, data security and warehousing logistics link information is inaccurate and the problems existing in the ${ }^{[7]}$. Therefore, the blockchain system based on logistics platform and blockchain technology can better solve problems such as logistics distribution, parcel settlement and parcel transmission [8], improve the utilization rate of resources, simplify the transaction process and greatly improve the overall efficiency of the industry.

\subsection{Application of blockchain technology in logistics finance.}

In recent years, logistics finance has been rapid development in our country, through the analysis of the principle characteristics of chain block, block chain technology can be used to build the commodity warehousing, trade, finance and sales of closed-loop chain, and to digital asset management, in order to realize the enterprise operating data and asset information accurate matching and control all the way, so as to ensure the authenticity of the asset information, transparency, and tamper-resistant ${ }^{[9]}$. An important feature of the chain block technique is not tamper with the sex, so we can be information goods are valued, capitalization, it can real-time trace all items in block chain system, goods can be verified at the same time, and all of the information data cannot be tampered with, thus fixed the sole ownership of the item. Therefore, the application of blockchain technology in the field of logistics finance can make the logistics goods have the characteristics of assets. On the other hand, with the help of the blockchain basic platform, funds can be more quickly and effectively connected to the logistics industry, which can help solve the financing problems of small and micro-sized enterprises in the logistics supply chain and accelerate the expansion and development of small and medium-sized enterprises.

\section{Application of blockchain technology in building intelligent logistics tracking system}

\subsection{Smart logistics tracking system based on "blockchain technology.}

Traditionally, supply chain transactions are done largely by hand, so the risk of recording errors and delays is higher, which can lead to differences between what is recorded and what is actually loaded. And blockchain, as a Shared distributed transaction ledger, will help keep track of all transactions and asset flows in the business network. According to the characteristics of decentralization, de-trust, tampering and traceability of blockchain, we can build a blockchain system exclusive to logistics transportation based on logistics platform and blockchain technology 
to realize intelligent tracking of goods. That is to say, build the inherent structure from the existing "commodity origin - trading platform - terminal consumer", directly across the retail platform, digitize the end-to-end supply chain through blockchain and Internet of things, and achieve the point-to-point transaction from the origin to the terminal. For example, a sensor device is installed on the truck and the cargo package, and then the logistics information of the cargo transportation and distribution process can be obtained through the on-board sensor, and then the data information enters the blockchain to establish a single Shared repository. All participants in the system can then manage and monitor the truck's transportation route and the estimated arrival time of the goods, and the system will also send data records of the goods to users in real time. This will not only improve the transparency of commodity trading and data, but also better solve problems such as logistics transportation, parcel delivery and parcel settlement, and reduce costs such as human and material resources ${ }^{[10]}$.

\subsection{Analysis of intelligent logistics tracking scheme based on "blockchain technology.}

Maersk and IBM's blockchain logistics solutions. Today, about 90 percent of the world's merchandise trade is transported by sea. Therefore, to realize the management and real-time tracking of hundreds of millions of shipping containers is a very complicated work that costs much manpower, material resources and money. Although the use of artificial intelligence, robotics, Internet of things and other technologies can solve these problems to a large extent, they are still not perfect $^{\text {[11] }}$. Therefore, in 2017, IBM and maersk proposed a new logistics scheme, namely, based on the development of super ledgers, the block chain technology was applied to the tracking of maritime logistics, and the process of supply chain was digitized. The digital solution of global trade based on blockchain technology is applied to maritime logistics. The digital solution of global trade based on blockchain technology is applied to maritime logistics, which can simplify the transportation of goods to the greatest extent. All enterprises and individuals in the blockchain can track the transportation of goods.

\section{Conclusion}

Based on block chain technology and logistics platform to create a set of exclusive in logistics transportation intelligent tracking system, through the chain block and the Internet of things will end-to-end supply chain to the digital processing, use of chain blocks all have the traceability, tamper-proof, decentralized data storage way and time stamp to prove the existence of the characteristic such as, maintenance of data and transactions, records and certification. All participants in the system can manage and monitor the transportation routes of goods and the estimated arrival time of goods, verify the logistics information in real time, and realize the intelligent tracking of goods. Through the real-time monitoring of express parcels, the transparency of data and transactions can be improved, as well as the problems of parcel transportation, logistics distribution and parcel settlement can be better solved, and the cost of human and material resources can be reduced.

\section{Acknowledgment}

This work is supported by National Social Science Fund Project 15BJY017; this work is supported by Heilongjiang Provincial Social Science Fund Project "Research on the Construction of Heilongjiang Province Financial Sharing Platform Based on the Perspective of Blockchain Technology" (18JYE672).

\section{References}

[1] Zhang Liucheng. Application-oriented Institutes Innovation Thinking and Practice of Talent Training Mode [J]. Advances in Social Science, Education and Humanities Research, 2016(59), pp. 1425-1429 
[2] Zhang Liucheng. Analysis on the future development of cloud Education [J]. Advances in Social Science, Education and Humanities Research,2016(59), pp. 1477-1480

[3] Zhang Liucheng. Research on the construction of education service platform based on Cloud Computing [J]. Advances in Social Science, Education and Humanities Research, 2016(59), pp. 1481-1484

[4] Zhang Liucheng. Application Prospect of Block Chain Technology in Accounting Industry,2018 International Conference on Mechatronics Engineering and Computer Sciences(ICMECS 2018). IEEE, 2018:559-562.

[5] Zhang Liucheng, Chen Meizhi. The analysis of the deep processing industry of agricultural products and strategy in Heilongjiang Province,2018 International Conference on Mechatronics Engineering and Computer Sciences(ICMECS 2018). IEEE, 2018:568-572.

[6] Zhang Liucheng. Impact of Block-Chain Technology on Auditing, 2018 International Conference on Mechatronics Engineering and Computer Sciences (ICMECS 2018). IEEE, 2018:573-577.

[7] Zhang Liucheng. Data Reliability Research on Accounting Information System Based on Block Chain Technology, 2018 International Conference on Mechatronics Engineering and Computer Sciences (ICMECS 2018). IEEE,2018:604-607 